\title{
Guía de evidencia en el tratamiento de la bronquiolitis.
}

\author{
Revisión
}

Eduardo A. Lara-Pérez.

Hospital de Gineco-Pediatría No. 71, Instituto Mexicano del Seguro Social. Veracruz, Veracruz, México.

\section{RESUMEN.}

Introducción.- a pesar de la alta prevalencia de la bronquiolitis como padecimiento infeccioso, hasta el momento no existe un tratamiento especifico. Actualmente el tratamiento de la bronquiolitis se ha polemizado por la cantidad de recomendaciones. Por un lado; las terapias "clásicas" y por otro las terapias "novedosas"; ambas terapias resultan polémicas cuando se analizan científicamente. Objetivo.- Evaluar con base a una guía la terapéutica común o clásica y terapia actual de la bronquiolitis, con fundamentos creíbles o demostrables.

Material y método.- Concepto diagnóstico de bronquiolitis y score de evaluación de la bibliografía; nivel de evidencia, evaluación del trabajo y recomendación.

Resultados.- Se integran tablas de evaluación bibliografica de los trabajos y recomendaciones acerca de los tratamientos conocidos.

Discusión.- A pesar de lo común de algunos tratamientos de la bronquiolitis en los servicios pediátricos, estos tratamientos carecen o están limitados en el grado de demostración científica al evaluar un beneficio. Algunos tratamientos populares dentro del tratamiento de la bronquiolitis carecen de evidencia científica, a diferencia de otras terapias que automáticamente se descartan, pero no se ha demostrado tampoco que estas no sean útiles. Se recomienda realizar estudios aleatorizados que ofrecen un mejor respaldo científico.

(Rev Biomed 2002; 13:211-219)

Palabras clave: Bronquiolitis, tratamiento, guía de evidencia, análisis terapéutico.

\section{SUMMARY.}

Evidence guide for the treatment of bronchiolitis.

Introduction.- Despite de high prevalence of bronchiolitis as an infectious disease, until this moment there is no specific treatment. Nowadays there has been a lot of dispute about the treatment of bronchiolitis because of the quantity of recommendations; on one side; the "classic" therapies and on another the novel therapies, both therapies result to be polemic when they have been

Solicitud de sobretiros: Dr. Eduardo A. Lara-Pérez, Pedro de Alvarado 201, Frac. Reforma, C.P. 91919, Veracruz, Veracruz, México. 


\section{EA Lara-Pérez.}

scientifically analyzed.

Objective.- To evaluate based on a guide; the classic or common therapeutic and the actual therapy of bronchiolitis with demonstrable and credible foundations.

Material and Method.- Diagnostic concept of bronchiolitis and bibliography; score evaluation, Evidence level, work evaluation and recommendation.

Results.- Bibliographic evaluation charts of the works and recommendations about the known treatments are integrated.

Discussion.- despite the commoness of some bronchiolitis treatments in the pediatric services, these treatments lack or are limited in the degree of scientific demonstration of evaluating a benefit. Some popular treatments within the bronchiolitis treatment, have a lack of scientific evidence, unlike other therapies which are automatically ruled out, but it has not been proved that neither of these ones are not useful. It is recommended to realize random studies that offer a better scientific support.

(Rev Biomed 2002; 13:211-219)

Key words.- Bronchiolitis, evidence guide, therapeutic analysis.

\section{INTRODUCCIÓN.}

La infección respiratoria de las vías aéreas bajas mas frecuente en el lactante es la bronquiollitis (1), que ha despertado un gran interés en la pediatría por su alta incidencia y elevada morbimortalidad (1). Sin embargo, el tratamiento de la bronquiolitis ha cambiado poco a lo largo de los años. No existen tratamientos cuya efectividad se haya demostrado y por lo tanto, en muchos casos, la estrategia o plan terapéutico carece de evidencias (1).

El objetivo de esta revisión es "analizar" y "actualizar" las recomendaciones terapéuticas en la bronquiolitis aguda, basándonos en el nivel de evidencia actualmente existente. Lógicamente, las variables a considerar son múltiples y exceden la pretensión de este trabajo; por ello se han establecido acotaciones, centrando esta discusión en el paciente tipo: lactante menor de 12 meses, previamente sano, que padece un primer episodio "típico" de bronquiolitis aguda por virus "sincitial respiratorio": Deben excluirse los pacientes con patología previa (displasia broncopulmonar, inmunodeficiencias, cardiopatías, fibrosis quística), los ingresados a la (UCIP) unidad de cuidados intensivos pediátricos por síndrome de distrés respiratorio agudo (SDRA) secundario, los de mas de un año y los pacientes con segundo o ulterior episodio de bronquiolitis.

\section{Metodología empleada.}

Para cada tratamiento analizado nos hemos planteado las siguientes cuestiones:

1.- $₫$ En lactantes menores de 12 meses previamente sanos con un primer episodio típico de bronquiolitis aguda es útil el tratamiento con " $X$ " para conseguir una mejora sintomática expresada como la mejoría en su score clínico, y la disminución de sus requerimientos de oxigeno suplementario ?

2.- $₫$ En lactantes menores de 12 meses previamente sanos con un primer episodio típico de bronquiolitis aguda es útil el tratamiento con " $X$ " para mejorar el pronostico del paciente, expresado como el acortamiento de la estancia hospitalaria y la incidencia y frecuencia posterior de crisis de hiperreactividad bronquial?

Este trabajo lo hemos realizado siguiendo la estrategia empleada en la medicina basada en la evidencia para la valoración de tratamientos (2):

1.- Búsqueda bibliográfica.

2.- Determinación del "nivel de evidencia" de los trabajos seleccionados (cuadro 1).

3.- Evaluación critica de la calidad de cada trabajo. 4.- Integración de las evidencias en un tipo concreto de recomendación final (cuadro 2).

La revisión bibliográfica se ha realizado a través de Med-Line y la Cochrane Library, suplementándose con referencias adicionales localizadas a través de la bibliografía listada de artículos, siguiendo una estrategia de búsqueda

\section{Revista Biomédica}


Tratamiento de la Bronquiolitis.

Cuadro 1

Niveles de evidencia considerados y aplicados a los equipos seleccionados en la búsqueda bibliográfica.

\begin{tabular}{ll}
\hline $\begin{array}{l}\text { Nivel } \\
\text { evidencia }\end{array}$ & \multicolumn{1}{c}{ Definición } \\
\hline 1 & Estudios aleatorios controlados con muestras grandes y resultados significativos $(\mathrm{p}<0,05)$ \\
2 & Estudios aleatorios controlados con muestras pequeñas y/o con resultados neutros $(\mathrm{p}=\mathrm{ns})$ \\
3 & Estudios prospectivos observacionales no aleatorizados \\
4 & Estudios retrospectivos observacionales no aleatorizados \\
5 & Series de pacientes, opinión de expertos o consensos \\
6 & Experimentación animal \\
7 & Extrapolaciones razonables a partir de datos existentes o revisiones \\
8 & Conjeturas racionales, sentido común \\
\hline
\end{tabular}

Cuadro 2

Grados de recomendación: clasificación según el nivel de evidencia, significación e implicaciones prácticas.

\begin{tabular}{|c|c|c|c|}
\hline Clase & Evidencias & Revisión de consenso & Recomendación practica \\
\hline A & $\begin{array}{l}\text { Nivel optimo (1 o más } \\
\text { estudios de nivel } 1)\end{array}$ & $\begin{array}{l}\text { Evidencias excelentes, eficacia } \\
\text { probada, recomendación definitiva }\end{array}$ & $\begin{array}{l}\text { Siempre aceptable, } \\
\text { seguridad probada definitivamente }\end{array}$ \\
\hline B & Nivel elevado-bueno & Evidencias buenas, aceptable y útil & $\begin{array}{l}\text { Aceptable, segura y útil, posible } \\
\text { "tratamiento de elección" }\end{array}$ \\
\hline $\mathrm{C}$ & Nivel regular-bajo & Evidencias regulares, aceptable y útil & $\begin{array}{l}\text { Aceptable, segura y útil, posible } \\
\text { "tratamiento alternativo" }\end{array}$ \\
\hline $\mathrm{D}$ & $\begin{array}{l}\text { Alguna evidencia, } \\
\text { incompleta o con } \\
\text { resultados contradictorios }\end{array}$ & $\begin{array}{l}\text { Evidencia limitada para } \\
\text { establecer recomendación }\end{array}$ & Aplicable reconociendo sus limitaciones \\
\hline $\mathrm{E}$ & $\begin{array}{l}\text { No evidencia positiva } \\
\text { o evidencia de efecto } \\
\text { perjudicial }\end{array}$ & $\begin{array}{l}\text { Inaceptable, carente de beneficio, } \\
\text { puede ser peligrosa }\end{array}$ & No recomendada \\
\hline
\end{tabular}

estándar (2). Todos los artículos seleccionados fueron clasificados en niveles de evidencia de 1 a 8 (de mayor a menor evidencia) (cuadro 1) y posteriormente analizados críticamente según su calidad y fuerza metodológicas. Posteriormente, se han integrado en una recomendación práctica final, graduada de A a E (de "mejor" a "peor" nivel) (cuadro 2).

\section{Tratamiento de soporte.}

El manejo terapéutico básico de una bronquiolitis aguda típica se centra en asegurar la oxigenación a hidratación del paciente, administrando oxigeno suplementario en los pacientes hipóxicos (saturación arterial de oxigeno $<92 \%$ ) y garantizando una adecuada hidratación, si es posible por vía oral/enteral o en su defecto por vía parenteral. Estas medidas, junto con una monitorización adecuada, constituyen el tratamiento de soporte de uso generalizado y uniformemente aceptado en el manejo de estos pacientes $(3,5)$. Las evidencias directas en que se apoya esta terapia son escasas, siendo la mayoría extrapolaciones de datos obtenidos en otras patologías, perpetuadas en sucesivas revisiones y consensos de expertos (estudios de nivel 6 y 7). Sin embargo, la imposibilidad ética de realizar actualmente estudios aleatorios controlados (EAC) sobre estos tratamientos, mantiene de forma consensuada el grado B de recomendación para esta práctica.

Vol. 13/No. 3/Julio-Septiembre, 2002 


\section{EA Lara-Pérez.}

Cuadro 3

Resumen de los grados de recomendación de los diferentes tratamientos teóricamente aplicables en un primer episodio de bronquiolitis aguda en un lactante previamente sano, basados en los niveles de evidencia actualmente existentes.

\begin{tabular}{ll} 
Intervención terapéutica & $\begin{array}{c}\text { Grado de } \\
\text { recomendación }\end{array}$ \\
\hline Soporte: monitorización + oxigeno + hidratación & $\mathrm{B}$ \\
Fisioterapia respiratoria & $\mathrm{D}^{*}$ \\
Nebulización/humidificación & $\mathrm{D}^{*}$ \\
Broncodilatadores & \\
- Beta agonistas & $\mathrm{E}$ \\
- Adrenérgicos & $\mathrm{B}^{*}$ \\
- Anticolinérgicos & $\mathrm{D}^{*}$ \\
Corticosteroides & $\mathrm{E}$ \\
Antibióticos & $\mathrm{E}$ \\
Ribavirina & $\mathrm{E}$ \\
Heliox & $\mathrm{C} *$ \\
Inmunoglobulinas & $\mathrm{E}$ \\
Anticuerpos monoclonales & $\mathrm{D}^{*}$ \\
Vitamina A & $\mathrm{E}$ \\
Hierbas chinas (Shuang Huang Lian) & $\mathrm{D}^{*}$ \\
Interferón & $\mathrm{E}$ \\
Xantinas & $\mathrm{E}$ \\
\hline
\end{tabular}

* Intervenciones terapéuticas que podrían mejorar su nivel de recomendación si se acumularan nuevas evidencias con EAC de alta calidad.

\section{Humidificación y nebulización templada.}

A pesar de uso prácticamente generalizado, sorprendentemente existen pocos estudios que evalúen el papel de la nebulización/humidificación templada en el tratamiento de la bronquiolitis aguda $(1,4,6)$. No se ha demostrado que la nebulización/ humidificación templada sea beneficiosa. No existen evidencias de que la humidificación en sí misma tenga efecto positivo alguno en estos pacientes, entre otras razones porque son pocas las gotas que alcanzan el tracto respiratorio inferior, donde se espera actué licuando las secreciones. Por otra parte, no está muy claro si la humidificación es totalmente inocua en estos pacientes. La escasez de evidencias, la eventual posibilidad de efectos adversos, y su inclusión repetida en revisiones y consensos de expertos, mantienen a nuestro juicio el nivel de recomendación clase D para esta práctica, a la espera de estudios de nivel 1 que definan con mayor precisión su papel.

\section{Fisioterapia.}

A pesar de su uso rutinario, no existen evidencias directas que demuestren que la fisioterapia respiratoria sea beneficiosa en estos pacientes (7). Por lo tanto, el grado de recomendación actual de esta practica es clase D, y sería interesante la realización de EAC con muestras grandes para elucidar definitivamente su papel.

\section{Broncodilatadores. \\ Beta-2 agonistas}

Existen dos revisiones sistemáticas (RS) sobre los broncodilatadores en el tratamiento de la bronquiolitis: la de Kellner (8) que recoge 8 EAC con un total de 485 niños y la de Flores (9) que incluye $5 \mathrm{EAC}$ con un total de 251 niños. Ambos meta-análisis demuestran como los broncodilatadores mejoran directamente los scores clínicos de niños con bronquiolitis leve a moderada, aunque no producen mejoría significativa en la saturación de oxigeno ni disminuye el porcentaje y duración de ingresos.

Hay que reseñar en el trabajo de Kellner (8), la heterogeneidad de los EAC incluidos, que incluye además de los beta- 2 agonistas, agentes adrenérgicos y anticolinérgicos; aun así, la mejoría global en el score calculada incluyendo todos los EAC, es mínima como para que tenga importancia clínica real. El subgrupo de EAC donde el efecto beneficioso era más marcado, era también metodológicamente más débil, sobre todo por la inclusión indiscriminada de pacientes con sibilancias recurrentes mayores de un año (asmáticos). La RS de Flores (9), únicamente incluye beta-2 agonistas, y sólo detecta una mejoría mínima en la saturación de oxígeno y en la frecuencia cardiaca, sin cambios en la frecuencia respiratoria y la tasa y la duración de hospitalización. Además, los broncodilatadores pueden mejorar la "apariencia" clínica del niño a

\section{Revista Biomédica}


Tratamiento de la Bronquiolitis.

través de un efecto estimulante general no relacionado con sus efectos sobre la función respiratoria (10), ni tampoco podemos olvidar las evidencias nivel 1 y 2 que demuestran la existencia de múltiples efectos adversos derivados del uso de beta- 2 agonistas en estos pacientes, incluyendo crisis hipóxicas (11). Por todo ello, los beta-2 agonistas no deben ser utilizados de forma rutinaria en el tratamiento del primer episodio de bronquiolitis aguda de lactantes previamente sanos (recomendación clase E). Su posible utilidad en la subpoblación especifica de pacientes, mayores de 6 meses, con marcada hiperreactividad bronquial y/o sibilancias recurrentes y/o riesgo de desarrollo de asma, está por determinar (recomendación clase D), máxime cuando se trata de una población de difícil identificación. En cualquier caso, si se utiliza el beta- 2 agonista, este debe ser suspendido si el plazo de 60 min de su aplicación no se objetiva mejoría alguna. A pesar de estas evidencias, el uso de beta-2 agonistas en este contexto clínico continua siendo una práctica muy extendida, cercana al $80 \%{ }^{4}$, y además, rara vez se retira aunque se haya comprobado que no ejerce mejoría alguna en el paciente (12).

\section{Adrenérgicos.}

Las evidencias existentes sugieren que la adrenalina es muy superior a los beta- 2 agonistas puros en el tratamiento de la bronquiolitis aguda, sin presentar efectos secundarios relevantes, según resultados basados en la mejoría del score clínico y de la oxigenación (13), su beneficio sobre la función respiratoria (14) y la disminución en el porcentaje de ingresos (13). Aunque es posible que sean necesarios estudios que evalúen más claramente su papel a medio y largo plazo, en el momento actual, la adrenalina constituye el agente broncodilatador de elección en el tratamiento de la bronquiolitis aguda y una opción terapéutica clase B en estos pacientes.

\section{Anticolinérgicos.}

Los pocos trabajos existentes sobre el uso de agentes anticolinérgicos, solos o en combinación con beta- 2 agonistas, en el tratamiento de la bronquiolitis aguda no encuentran efectos beneficiosos (15). Una RS reciente (6 EAC) sobre el papel de los agentes anticolinérgicos (bromuro de ipatropio) en el tratamiento de lactantes "sibilantes" menores de 2 años, excluyendo los afectados de bronquiolitis aguda, no encontró diferencias frente al placebo ni en la respuesta clínica inicial en urgencias (saturación de oxigeno, frecuencia respiratoria) ni en la duración del ingreso (16). Teniendo en cuenta que los EAC existentes con escasos y de baja calidad, creemos que mientras no se realicen nuevos estudios específicos, el nivel de recomendación de anticolinérgicos en nuestra población de estudio es clase D.

\section{Corticosteroides.}

Existen múltiples RS y EAC de alta calidad (nivel 1) que demuestran que los corticoides, administrados por vía sistémica o inhalados, no proporcionan beneficios ni durante la fase aguda ni en la evolución posterior de los lactantes previamente sanos con un primer episodio de bronquiolitis aguda, presentando además efectos adversos bien documentados (nivel 1) tales como la hiperglucemia y/o la inmunosupresión $(17,18)$. Por el contrario hay algunos trabajos entre los que destaca un metaanálisis realizado por Garrison y col (19), que encuentran efectos positivos frente a placebo a nivel sintomático, y una discreta disminución en la duración de síntomas y estancia media; sin embargo, los EAC incluidos son heterogéneos, no se excluyeron pacientes con historia previa de sibilancias, las escalas clínicas utilizadas y en concreto la valoración de cambios en la oximetría eran muy variables, y la mejoría media de 1.6 en el score a las 24 h tiene un valor discutible, tanto por el modo de cálculo como por su significación. Por tanto, en el momento actual la recomendación general basada en la evidencia del uso de corticosteroides en los niños con bronquiolitis aguda es de clase E. A pesar de ello y en contra de las evidencias, en el año 97 todavía 


\section{EA Lara-Pérez.}

se administraban corticoides hasta en un $80 \%$ de los casos (4), y en estimaciones más recientes el porcentaje aun se sitúa por encima del $50 \%$ (3).

\section{Antibióticos.}

Se ha estimado que entre un 57 a un $81 \%$ de los lactantes diagnosticados de bronquiolitis reciben antibióticos (20). Sin embargo, el uso rutinario de antibióticos carece de efectos beneficiosos, no sólo en la bronquiolitis sino también en la neumonía por VRS (nivel 1) (21). Existe incluso alguna evidencia de que la infección por VRS no predispone a la sobreinfección bacteriana (nivel 3) (22). Por tanto no está recomendado su uso sistemático en estos pacientes (recomendación clase E). Estando su indicación únicamente justificada ante la presencia de un foco bacteriano documentado (recomendación clase B): otitis media aguda (miringitis con/sin abombamiento con/sin otorrea), sinusitis (descarga nasal purulenta prolongada $(>10$ días) con/sin corroboración radiológica), o neumonía (hallazgos radiológicos positivos con negatividad de estudios virales) (20).

\section{Ribavirina.}

No existen evidencias claras de que la utilización de Ribavirina pueda mejorar clínicamente y/o reducir la mortalidad de nuestra población de estudio tal como se deduce de la RS de 8 EAC realizada por Randolph y col (23), si bien es una RS heterogénea de EAC de muestra pequeña y baja calidad. Un EAC posterior (nivel 2) (24) demostraba que la Ribavirina era también ineficaz en la disminución de la duración de la ventilación mecánica y/o el tiempo de estancia de pacientes con bronquiolitis grave que requieren intubación. Menos claro esta su papel sobre la morbilidad a largo plazo: un estudio de Edell y col (25) muestra que su administración frente a placebo en lactantes previamente sanos con bronquiolitis reduce significativamente la incidencia y frecuencia de episodios posteriores de hiperreactividad bronquial aguda, aunque se trata de un trabajo de nivel 4, con una muestra pequeña y seguimiento <
$80 \%$, por tanto de valor muy limitado. Por otro lado existen trabajos de potencia metodológica similar que no encuentran estas diferencias. Por tanto, el cuerpo de evidencia existente nos permite establecer una recomendación general clase E sobre el uso de Ribavirina en estos pacientes, y una recomendación clase $\mathrm{D}$ sobre su uso en pacientes con bronquiolitis de alto riesgo y/o bronquiolitis severa, siendo necesarios EAC de alta calidad que clarifiquen su papel en este ultimo grupo de pacientes.

\section{Heliox.}

El heliox, una mezcla gaseoso de helio y oxigeno de menor densidad que el aire. Se ha empleado con éxito en el tratamiento sintomático de múltiples enfermedades respiratorias de tipo obstructivo. Hollman y col (26), realizaron un EAC en el que observan que su aplicación mejoraba el estado respiratorio de pacientes con bronquiolitis aguda leve o moderada, y de forma más marcada en los niños con mayor compromiso respiratorio; sin embargo, aunque se trata de EAC nivel 2, la muestra es pequeña y no homogénea, parte de los pacientes no fueron aleatorizados, y no se consideraron los efectos a medio y largo plazo. Se ha realizado un estudio prospectivo no aleatorizado (datos no publicados), en el que se observa como la administración de heliox a lactantes previamente sanos con bronquiolitis aguda, fue capaz de mejorar significativamente el estado respiratorio de los pacientes sobre la base de su score clínico y la reducción de la taquipnea y taquicardia asociadas, comparado con los pacientes que recibieron únicamente tratamiento convencional. Basándonos en las evidencias existentes en el momento actual, sólo es posible establecer una recomendación clase C para el tratamiento con heliox de la bronquiolitis, siendo necesarios EAC de alta calidad que clarifiquen el potencial de este tratamiento.

\section{Inmunoterapia.}

La inmunoterapia frente a VRS (IVRS) y los anticuerpos monoclonales humanizados frente al

\section{Revista Biomédica}




\section{Tratamiento de la Bronquiolitis.}

VRS (palivizumab) tienen una eficacia probada en la prevención de la infección aguda en lactantes de alto riesgo, pero su papel en la infección ya establecida ha sido poco explorado. Diversos EAC muestran como tanto la IVRS, como la gammaglobulina inespecífica intravenosa o en aerosol carecen de eficacia en el tratamiento de la bronquiolitis aguda de lactantes previamente sanos en términos de duración de estancia hospitalaria, duración de tiempo de ventilación mecánica o duración del tratamiento con oxígeno suplementario, siendo la recomendación sobre su uso clase E $(27,29)$. Existen evidencias limitadas de la utilidad de IVRS en los pacientes de alto riesgo y/o con bronquiolitis grave que precisan de ventilación mecánica, con una discreta tendencia a disminuir el tiempo de estancia en UCIP (29). En el momento actual la recomendación de su uso en este subgrupo es clase D. Respecto al palivizumab, al margen de efectos positivos detectados en estudios nivel 6, sólo hemos encontrado un estudio nivel 1 de muestra pequeña en niños ventilados mecánicamente por infección por VRS en el que se observa una disminución en la concentración de VRS en el aspirado traqueal frente al grupo placebo, sin diferencias en clínica, tiempo de ventilación y estancia (30). Son necesarios más EAC con palivizumab en infección establecida por VRS, estableciendo mientras tanto una recomendación clase D.

\section{Vitamina A.}

La detección de niveles séricos anormalmente bajos de vitamina A en lactantes con bronquiolitis por VRS, originó diversos trabajos nivel 1 sobre su suplementación terapéutica, que sin embargo, demostraron una ausencia total de beneficios de dicha práctica, e incluso una prolongación del tiempo de estancia media (31). Por tanto, la vitamina A no tiene lugar en el esquema terapéutico de la bronquiolitis aguda (recomendación clase E).

Hierbas medicas chinas (Shuang Huang Lian). Existen algunas evidencias aisladas (nivel 2 y
3) de poca calidad metodológica, que sostienen que la administración oral, parenteral y sobre todo en aerosol, del tratamiento herbal chino Shuang Huang Lian, mejora sintomáticamente a los pacientes con bronquiolitis aguda y disminuye su estancia media hospitalaria, basándose en las propiedades virostáticas e inductoras de producción de interferón de estas sustancias (32). En el momento actual, la recomendación es clase $\mathrm{D}$, a la espera de nuevas evidencias que delimiten mejor su papel/clarifiquen su potencial.

\section{Interferón.}

La producción de interferón está disminuida durante la infección por VRS. La evidencia actual (estudios nivel 2 y 4), no encuentra beneficio de su administración intramuscular o en aerosol (recomendación clase E) (33).

\section{Xantinas.}

Los EAC existentes, entre los que destaca el de Labbe (34), no se encuentran efectos beneficiosos en la administración de teofilina a estos pacientes (recomendación clase E).

\section{Otros tratamientos.}

Existen otros tratamientos en los que las evidencias existentes son anecdóticas o claramente insuficientes para establecer una recomendación en el momento actual, pero en las que nuevos estudios podrían clarificar su papel. Entre ellas destacamos: furosemida nebulizada (Van Bever, 1995), dimetil sulfóxido (Zúñiga, 1975), mucolíticos (nacetilcisteína o ADNsa reconbinante), y Ketamina (Youssef-Ahmed, 1996).

\section{CONCLUSIONES.}

A pesar de la existencia de evidencias definitivas de que tratamientos "clásicos" empleados en la bronquiolitis aguda como los beta2 agonistas o corticosteroides son poco o nada eficaces, su uso continua siendo frecuente en México (cuadro 3).

Vol. 13/No. 3/Julio-Septiembre, 2002 


\section{EA Lara-Pérez.}

Por otro lado, otros tratamientos considerados "alternativos" tales como la adrenalina, o el heliox han demostrado ser beneficiosos y deberían realizarse esfuerzos para obtener evidencias científicas que confirmen o descarten su utilidad en este terreno.

\section{REFERENCIAS.}

1.- Everard ML. Bronchiolitis. Origins and optimal management. Drugs. 1995;49:885-96.

2.- Sitio web: Centres for Health Evidence.net en: .

3.- Pilar-Orive FJ, Casado-Flores J, García-Teresa MA. Infecciones respiratorias agudas en Unidades de Cuidados Intensivos Pediátricos. Estudio prospectivo multicéntrico. An Esp Pediatr 1998; 48:138-42.

4.- Kimpen JL, Schaad UB. Treatment of respiratory syncytial virus bronchiolitis: 1995 poll of members of European Society for Paediatric Infections Disease. Pediatr Infect Dis J 1997; 16:479-81.

5.- Lugo RA, Nahata M. Pathogenesis and treatment of bronchiolitis. Clin Pharm 1993; 12:95-116.

6.- Makela MJ, Ruuskanen O, Ogra PL. Treatment of respiratory syncytial virus infections in children. Ann Med 1994; 26:341-3.

7.- Webb MS, Martin JA, Cartlidge PH, Ng YK, Wright NA. Chest physiotherapy in acute bronchiolitis. Arch Dis Child 1985; 60:1078-9.

8.- Kellner VG, Rodríguez W, Kim HW. Intravenous immunoglobulin treatment of respiratory syncytial virus infections in infants and young children. Antimicrob Agents Chemother 1987; 31:1882-6.

9.- Flores G, Horwitz RI. Efficacy of beta 2-agonist in bronchiolitis: A reappraisal and meta-analysis. Pediatrics 1997; 100:233-9.

10.- Gadomsky AM, Lichenstein R, Horton L, King J, Keane V, Permutt T. Efficacy of albuterol in the management of bronchiolitis. Pediatrics 1994; 93:907-12.

11.- Ho L, Collins G, Landau LI, Le Souef P. Effect of salbutamol on oxygen saturation in bronchiolitis. Arch Dis Child 1991; 66:1061-4.

\section{Revista Biomédica}

12.- Lugo RA, Salyer JW, Dean JM. Albuterol in acute bronchiolitis continued therapy despite poor response? Pharmocotherapy 1998: 198-202.

13.- Menon K, Sutcliffe T, Klasen TP. A randomized trial comparing the efficacy of epinephrine with salbutamol in the treatment for bronchiolitis. J Pediatr 1991; 126:10047.

14.- Lodrup-Carlsen KC, Carlsen KH. Inhaled nebulized adrenaline improves lung function in infants with acute bronchiolitis. Respir Med 2000; 94:709-14.

15.- Sammartino LP, Lines D. Efficacy of ipratropium bromide by metered dose aerosol and aerochamber in acute paediatric bronchiolitis. J Paediatr Child Health 1997; 33:459.

16.- Everard ML, Bara A, Kurian M. Anticholinergic drugs for wheeze in children under the age of two years (Cochrane Review). En: The Cochrane Library, Issue 1, Oxford: Update Software; 2001.

17.- Richter H, Seddon P. Early nebulized budesonide in the treatment of Bronchiolitis and the prevention of postbronchiolitic wheezing. J Pediatr 1998; 132: 849-53.

18.- Bulow SM, Nir M, Levin E. Prednisolone treatment for respiratory syncytial virus infection: a randomized controlled trial of 147 infants. Pediatrics 1999; 104:77.

19.- Garrison MM, Christakis DA, Harvey E, Cummins P, Davis RL. Systemic corticosteroids in infant bronchiolitis: A meta-analysis. Pediatrics 2000; 105:849.

20.- Henderson M, Rubin E. Misuse of antimicrobials in children with asthma and bronchiolitis. Pediatr Infect Dis J 2001; 20:214-5.

21.- Früs B, Andersen P, Brenöe E. Antibiotic treatment of pneumonia and Bronchiolitis: a prospective randomized study. Arch Dis Child 1984; 59:2038-45.

22.- Hall CB, Powell KR, Schnabel KC. Risk of secondary bacterial infection in infants hospitalized with RSV infection. J Pediatr 1998; 113:266-71.

23.- Randolph AG, Wang EEL. Ribavirin for respiratory syncytial virus lower respiratory tract infection. In: The Cochrane Library, Issue 1. Oxford: Update Software; 2000.

24.- Guerguerian AM, Gauthier M, Lebel MH, Farrell CA, Lacroix J. Ribavirin in ventilated respiratory syncytial virus 
bronchiolitis. Am J Respir Crit Care Med 1999; 160:82934.

25.- Edell D, Bruce E, Hale K, Khoshoo V. Reduced Longterm respiratory morbidity after treatment of respiratory syncytial virus bronchiolitis with Ribavirin in previously healthy infants: a preliminary report. Pediatr Pulmunology $1998 ; 25: 154-8$.

26.- Hollman G, Shen G, Zeng L. Helium-oxygen improves clinical asthma scores in children with acute bronchiolitis. Crit Care Med 1998; 26:1731-6.

27.- Wang EEL, Tang NK. Immunoglobulin for preventing respiratory syncytial virus infection (Cochrane Review): In: The Cochrane Library, Issue 1. Oxford: Update Software; 2001.

28.- Hemming VG, Rodriguez W, Kim HW. Intravenous immunoglobulin treatment of respiratory syncytial virus infection in infants and young children. Antimicrob Agents Chemother 1987; 31:1882-6.

29.- Rodriguez WJ, Gruber WC, Groothuis JR. Respiratory syncytial virus immune globulin treatment of RSV lower respiratory tract infection in previously healthy children. Pediatrics 1997; 100:937-42.

30.- Malley R, DeVincenzo J, Ramilo O. Reduction of respiratory syncytial virus (RSV) in tracheal aspirates in intubated infants by use of humanized monoclonal antibody to RSV F protein. J Infect Dis 1998; 178:1555-61.

31.- Kjolhede CL, Chew FJ, Gadonsky AM, Marroquin DP. Clinical trial of vitamin A as adjuvant treatment for lower respiratory tract infections. J Pediatr 1995; 126: 80712.

32.- Liu C, Douglas RM. Chinese herbal medicines in the treatment of acute respiratory infections: a review of randomized controlled clinical trials. Med J Aust 1998; 169:579-82.

33.- Chipps BE, Sullivan WF, Portnoy JM. Alpha-2a interferon for treatment of bronchiolitis caused by respiratory syncytial virus. Pediatr Infect Dis J 1993; 12: 653-8.

34.- Labbe A, Billet P, Paire M, Storme B. Treatment of acute bronchiolitis in infants by oral suspensión theophylline. Double-blind study in 62 children. Pediatrics 1985; 40:195-9. 